\title{
Studies of regional adipose transplantation reveal a unique and beneficial interaction between subcutaneous adipose tissue and the intra-abdominal compartment
}

\author{
S. L. Hocking • D. J. Chisholm • D. E. James
}

Received: 21 December 2007 / Accepted: 7 February 2008/Published online: 14 March 2008

(C) Springer-Verlag 2008

To the Editor: Obesity is associated with insulin resistance and type 2 diabetes, with accumulation of intra-abdominal fat carrying a more severe disease risk than accumulation of subcutaneous fat. It remains unclear whether increased visceral fat has an adverse metabolic effect due to its location or to the unique properties of intra-abdominal adipocytes. Konrad et al. [1] reported that increasing intra-abdominal fat mass by transplantation of epididymal fat from normal mice into lean recipients improved fasting glucose tolerance and insulin sensitivity, achieving an effect opposite to the expected metabolic consequence of increased intra-abdominal fat. This suggests that obesity-induced alterations in adipose tissue function rather than mass are responsible for the adverse metabolic consequences of obesity. We hypothesised that the intrinsic properties of adipocytes are responsible for their metabolic effects, irrespective of their anatomical location. We have addressed this using regional adipose tissue cross-transplantation in which subcutaneous (inguinal) and intra-abdominal (epididymal) fat pads from donor mice were transplanted into the subcutaneous (group 1) or intra-abdominal (group 2) compartment of recipient mice on a high-fat diet. Our studies revealed that transplantation of intra-abdominal fat into either the intraabdominal or subcutaneous space had no effect on the metabolism of a recipient animal, whereas transplantation of subcutaneous fat into the intra-abdominal space had a significant protective effect on adiposity, insulin sensitivity and glucose tolerance.

\footnotetext{
S. L. Hocking $\cdot$ D. J. Chisholm $•$ D. E. James $(\varangle)$

Diabetes and Obesity Program,

Garvan Institute of Medical Research,

384 Victoria St.,

Darlinghurst, NSW 2010, Australia

e-mail: d.james@garvan.org.au
}

In our experiments mice had free access to a high-fat diet (45\% of energy as fat, $20 \%$ as protein, $35 \%$ as carbohydrates, 19.7 kJ/g, [based on rodent diet no. D12451; Research Diets, New Brunswick, NJ]) commencing 1 week prior to surgery and continued for the study duration. Transplantation was performed at 7 weeks. For transplants into the subcutaneous compartment, grafts were implanted through small incisions in the back as previously described [2]; for intra-abdominal transplantation, grafts were sutured to the visceral side of the peritoneum on the anterior abdominal wall [1]. Sham operated mice received identical surgical treatment without transplant. Glucose tolerance tests were performed 12 weeks after transplantation [3] and body composition was determined when mice were killed (13 weeks after transplantation). Experiments were carried out with approval from the Garvan Institute/St. Vincent's Hospital Animal Ethics Committee.

Equivalent amounts of subcutaneous and epididymal adipose tissue were transplanted in groups 1 and 2 (328 \pm 20 vs $326 \pm 10 \mathrm{mg})$. At death adipose grafts were clearly identified in both depots and appeared viable with evidence of angiogenesis. Histologically, grafts retained features of their respective endogenous beds comprising well-defined adipocytes interspersed with stromovascular cells. There was no difference in weight gain in mice receiving transplants and sham operations in both groups (Table 1). Strikingly, mice receiving subcutaneous transplants into the intra-abdominal compartment displayed a significant reduction in the mass of various endogenous white adipose tissue beds compared with controls (Table 1). Inguinal, epididymal and retroperitoneal beds were reduced by 26, 33 and $35 \%$ respectively in these mice. This effect did not achieve significance in any of the other groups. No difference was seen in interscapular fat pad size in mice receiving subcutaneous transplants into the intra-abdominal compart- 
Table 1 Total body weight, fat depot weights and weight of harvested and implanted transplants

\begin{tabular}{|c|c|c|c|c|c|c|c|c|}
\hline & \multicolumn{4}{|c|}{ Subcutaneous space } & \multicolumn{4}{|c|}{ Intra-abdominal space } \\
\hline & $\begin{array}{l}\text { Sham } \\
(n=4)\end{array}$ & $\begin{array}{l}\text { SC graft } \\
\text { recipients } \\
(n=7)\end{array}$ & $\begin{array}{l}\text { AB graft } \\
\text { recipients } \\
(n=5)\end{array}$ & $\begin{array}{l}p \text { value } \\
\text { (ANOVA) }\end{array}$ & $\begin{array}{l}\text { Sham } \\
(n=6)\end{array}$ & $\begin{array}{l}\text { SC graft } \\
\text { recipients } \\
(n=6)\end{array}$ & $\begin{array}{l}\text { AB graft } \\
\text { recipients } \\
(n=6)\end{array}$ & $\begin{array}{l}p \text { value } \\
\text { (ANOVA) }\end{array}$ \\
\hline Initial weight (g) & $22.1 \pm 0.8$ & $21.1 \pm 0.5$ & $21.3 \pm 0.4$ & 0.43 & $21.5 \pm 0.6$ & $21.4 \pm 0.5$ & $21.7 \pm 0.5$ & 0.91 \\
\hline Final weight $(\mathrm{g})$ & $31.0 \pm 2.8$ & $29.4 \pm 0.9$ & $29.0 \pm 0.8$ & 0.67 & $29.4 \pm 1.3$ & $28.5 \pm 1.0$ & $29.1 \pm 1.3$ & 0.86 \\
\hline \multicolumn{9}{|l|}{ Fat depots (mg) } \\
\hline Inguinal & $828 \pm 266$ & $756 \pm 87$ & $689 \pm 44$ & 0.80 & $757 \pm 53$ & $558 \pm 49^{\mathrm{a}}$ & $631 \pm 87$ & 0.13 \\
\hline Epididymal & $1244 \pm 341$ & $990 \pm 115$ & $986 \pm 46$ & 0.54 & $1156 \pm 105$ & $776 \pm 101^{b}$ & $975 \pm 157$ & 0.13 \\
\hline Retroperitoneal & $416 \pm 113$ & $376 \pm 52$ & $323 \pm 23$ & 0.64 & $367 \pm 11$ & $239 \pm 29^{c}$ & $326 \pm 54$ & 0.07 \\
\hline Interscapular & $286 \pm 61$ & $248 \pm 18$ & $253 \pm 12$ & 0.67 & $257 \pm 14$ & $215 \pm 22$ & $222 \pm 23$ & 0.30 \\
\hline Removed transplant & & $307 \pm 33$ & $440 \pm 28$ & 0.02 & & $175 \pm 13$ & $380 \pm 30$ & 0.0001 \\
\hline Implanted transplant & & $312 \pm 33$ & $350 \pm 11$ & 0.37 & & $320 \pm 16$ & $332 \pm 13$ & 0.57 \\
\hline ipGTT AUC $(\mathrm{mmol} / 1 \times \min )$ & 1,998 & 1,764 & 1,741 & 0.28 & 1,980 & $1,495^{\mathrm{d}}$ & 1,815 & 0.04 \\
\hline
\end{tabular}

Values are means \pm SEM

${ }^{\mathrm{a}} p=0.02$ vs sham

${ }^{\mathrm{b}} p=0.03$ vs sham

${ }^{\mathrm{c}} p=0.002$ vs sham

d $p=0.008$ vs sham (Student's $t$ test)

ipGTT, intraperitoneal glucose tolerance test

ment and sham-controls, suggesting that the reduction in size of endogenous fat pads was specific to white adipose tissue. Although donor tissue was weight matched at the time of surgery, subcutaneous fat grafts reduced by $45 \%$ when transplanted into the intra-abdominal compartment and epididymal fat grafts increased by $25 \%$ when transplanted into the subcutaneous space (Table 1). Other fat grafts retained their original mass. Mice receiving subcutaneous transplants into the intra-abdominal compartment exhibited improved glucose tolerance compared with controls with a $25 \%$ decrease in the AUC for glucose $(p=0.008)$. This effect was not observed in the other groups.

Our finding that transplantation of epididymal fat into the intra-abdominal or subcutaneous space had no detrimental effects on metabolism is consistent with the findings of Konrad et al. [1]. Collectively, these findings argue against an effect of increased intra-abdominal fat mass per se in insulin resistance and provide further evidence that alterations in adipose tissue metabolism and physiology as a consequence of obesity are responsible for metabolic derangements.

Our results also demonstrate that transposition of subcutaneous fat into the intra-abdominal space creates some metabolic advantage for the animal, leading to reduced fat mass and improved glucose tolerance. The selectivity of this effect with regard to origin of donor tissue and site of transplant suggests that it is the unique combination of the fat depot and its location that confers this metabolic advantage. An observation reported here may provide novel insights. Subcutaneous transplants underwent a significant reduction in size when transposed into the intra-abdominal space and epididymal transplants significantly increased in size when transposed into the subcutaneous space. This may be due to a fortuitous mismatch between the origin and destination of the transplant. There is emerging evidence that angiogenic cells and innervation influence adipose tissue metabolism. A paracrine interaction between microvascular endothelial cells (MVECs) and pre-adipocytes has been observed which regulates adipose tissue growth [4, 5]. Adipose tissue is recognised to have autonomic innervation displaying somatotopy, with subcutaneous and intraabdominal fat pads innervated by separate sympathetic and parasympathetic neurons. Sympathetic input is catabolic and vagal input is anabolic [6]. It is feasible that a depot-specific cross-talk exists between adipocytes, MVECs and neurons and that this was disrupted by transposing subcutaneous and intra-abdominal fat into different compartments. It is postulated that alterations in adipocytes or stromovascular cells within the transplant generated, perhaps via alterations in adipokines, a systemic effect to reduce endogenous adipose tissue depots.

These findings raise new possibilities for the management of obesity. Autologous subcutaneous fat transplantation to the intra-abdominal space could, conceivably, confer a unique metabolic advantage.

Acknowledgments This work was supported by grants from the National Health and Medical Research Council of Australia, Diabetes Australia, and GlaxoSmithKline. We would like to thank J. Prins, Princess Alexandra Hospital Brisbane, for helpful advice and the Biological Testing Facility, Garvan Institute for animal care.

Duality of interest The authors declare that there is no duality of interest associated with this manuscript. 


\section{References}

1. Konrad D, Rudich A, Schoenle EJ (2007) Improved glucose tolerance in mice receiving intraperitoneal transplantation of normal fat tissue. Diabetologia 50:833-839

2. Gavrilova O, Marcus-Samuels B, Graham D et al (2000) Surgical implantation of adipose tissue reverses diabetes in lipoatrophic mice. J Clin Invest 105:271-278

3. Turner N, Bruce CR, Beale SM et al (2007) Excess lipid availability increases mitochondrial fatty acid oxidative capacity in muscle: evidence against a role for reduced fatty acid oxidation in lipid-induced insulin resistance in rodents. Diabetes 56:20852092

4. Hutley LJ, Herington AC, Shurety W et al (2001) Human adipose tissue endothelial cells promote preadipocyte proliferation. Am J Physiol Endocrinol Metab 281:E1037-E1044

5. Hutley L, Shurety W, Newell F et al (2004) Fibroblast growth factor 1: a key regulator of human adipogenesis. Diabetes 53:3097-3106

6. Kreier F, Fliers E, Voshol PJ et al (2002) Selective parasympathetic innervation of subcutaneous and intra-abdominal fat-functional implications. J Clin Invest 110:1243-1250 